\title{
Correlation between clinical parameters of crown and gingival morphology of anterior teeth and periodontal biotypes
}

\author{
Xiao-jie Yin ${ }^{1,2}$, Bang-yan Wei ${ }^{1}$, Xiu-ping Ke ${ }^{1}$, Ting Zhang ${ }^{1}$, Meng-yang Jiang ${ }^{1}$, Xia-yan Luo ${ }^{1}$ and Hui-qiang Sun ${ }^{1,2^{*}}$ (D)
}

\begin{abstract}
Background: In this study, we conducted a quantitative analysis of the clinical parameters of crown and gingival morphology (CGM) of the maxillary anterior teeth (MAT). We also analyzed the correlation of these parameters with periodontal biotype (PB), with a view to providing objective standards for PB diagnosis.

Methods: The three-dimensional (3D) maxillary digital models of 56 individuals were obtained using an intra-oral scanner. The following parameters were measured with the SpaceClaim software: gingival angle (GA), papilla width $(\mathrm{PW})$, papilla height $(\mathrm{PH})$, crown length $(\mathrm{CL})$, crown width $(\mathrm{CW})$, crown width/crown length ratio $(\mathrm{CW} / \mathrm{CL})$, buccolingual width of the crown (BLW), contact surface width (CSW), and contact surface height/crown length ratio (CS/ $\mathrm{CL}$ ). The PB were determined based on the transparency of the periodontal probe through the gingival sulcus. Independent factors influencing PB were analyzed by logistic regression, and the optimal cutoff values for the independent influencing factors were analyzed using receiver operating characteristic curves (ROC curves).

Results: There was no significant difference in the parameters of CGM of the MAT at the left and right sides. The thick biotype accounted for $69.6 \%$, and the parameters of $\mathrm{GA}, \mathrm{PW}, \mathrm{PH}, \mathrm{CW}, \mathrm{CW} / \mathrm{CL}$ and $\mathrm{CS} / \mathrm{CL}$ were significantly correlated with PB $(P \leq 0.2)$. GA (odds ratio $(O R)=1.206)$ and $P W(O R=5.048)$ were identified as independent predictive factors of $\mathrm{PB}$, with areas under the ROC curve (AUC) of 0.807 and 0.881 , respectively, and optimal cutoff values of $95.95^{\circ}$ and $10.01 \mathrm{~mm}$, respectively.

Conclusion: The CGMs of the MAT at the left and right side are symmetrical. The thin biotype accounts for a small proportion, and GA and PW are independent influencing factors of PB. GA of $95.95^{\circ}$ and PW of $10.01 \mathrm{~mm}$ are the optimal cutoff values for categorization of individuals as thick biotype. This indicates that when the GA and PW of the right maxillary central incisor are $G \geq 95.95^{\circ}$ and $\geq 10.01 \mathrm{~mm}$, respectively, there is a higher probability that these individuals will be categorized as thick biotype.
\end{abstract}

Keywords: Periodontal biotype, Gingival morphology, Crown morphology, Digital models, Cutoff value

\section{Background}

In 1989, Seibert and Lindhe [1] proposed the concept of $\mathrm{PB}$, that is, the thickness of the bucco-lingual gingiva can be divided into thick and thin biotypes. The CGM coordination and the stability of the gingival margin differ between different PBs, which directly influences the esthetic effect of restoration and patient satisfaction.

\footnotetext{
*Correspondence: whitedove69@163.com

'Department of Prosthodontics, School of Stomatology, Shandong University, Jinan 250012, Shandong, China

${ }^{2}$ Shandong Key Laboratory of Oral Tissue Regeneration \& Shandong Engineering Laboratory for Dental Materials and Oral Tissue Regeneration, Jinan, Shandong, China
}

(c) The Author(s). 2020 Open Access This article is distributed under the terms of the Creative Commons Attribution 4.0 International License (http://creativecommons.org/licenses/by/4.0/), which permits unrestricted use, distribution, and reproduction in any medium, provided you give appropriate credit to the original author(s) and the source, provide a link to the Creative Commons license, and indicate if changes were made. The Creative Commons Public Domain Dedication waiver (http://creativecommons.org/publicdomain/zero/1.0/) applies to the data made available in this article, unless otherwise stated. portant influence on the treatment effect and prognosis of periodontal surgery, plantation and orthodontics [2-4]. Therefore, correct classification of the PB is critically important in dental treatment.

In recent years, the correlation of $\mathrm{PB}$ with clinical parameters such as gingival thickness, crown morphology, and alveolar bone morphology has received increasing attention [5-12]. De Rouck et al. [5] measured intra-oral indexes including $\mathrm{CW} / \mathrm{CL}$, keratinized gingival width, and papilla height using calipers and a periodontal probe, and divided PBs into thin-scalloped biotype, 
thick-flat biotype and thick-scalloped biotype using the cluster analysis method. Stein et al. [10] conducted intra-oral measurements of keratinized gingival width and gingival thickness using a periodontal probe, measured the $\mathrm{CW} / \mathrm{CL}$ and papilla height using image analysis software, and explored the correlation of parameters such as gingival thickness and CW/CL. However, the correlation of PBs with maxillary margin and papilla width, in addition to the independent influencing factors of PBs, remain to be clarified.

The use of calipers and periodontal probes to measure the clinical parameters of gingival morphology are subject to the disadvantages of inconvenience and low accuracy. The results obtained using image analysis software are inclined to be influenced by the position of the head although most reports do not include a unified stipulation of the spatial position of head $[9,13]$. With the rapid development of computer technology, computer-aided design/computer-aided manufacturing (CAD/CAM) technology has been introduced for the esthetic restoration of anterior teeth. Lee et al. [14] analyzed the correlation between $\mathrm{PB}$ and the gingival papilla of the MAT using 3D digital models, while Wong et al. [15] explored the esthetic relationship between the incisal edge of the MAT and the upper border of the lower lip using 3D digital models.

In this study, we conducted a quantitative analysis of the clinical parameters of MAT, including GA, PW and $\mathrm{PH}$, using an intra-oral scanner and SpaceClaim software, with a view to providing a more accurate reference for the computer-aided esthetic analysis and design of anterior teeth. The transparency of the periodontal probe through the gingival sulcus was used for periodontal biotyping, thereby exploring the correlation of the $\mathrm{PB}$ of the right maxillary central incisor with the clinical parameters of the gingiva and crown, and analyzing the cutoff value of independent influencing factors with the aim of providing objective standards for periodontal biotyping.

\section{Methods}

\section{Study participants and inclusion criteria}

From January 2018 to June 2018, 56 study participants (13 males and 43 females) were selected from the oncampus students and young nurses from the School of Stomatology of Shandong University (China). The participants were Han nationality and the average age was $23.6 \pm 2.8$ years. The following inclusion criteria were applied: 1 ) the MAT and the maxillary first molar on both sides were in orderly alignment and had no anodontia, interspersed diastema, wedge-shaped defect, dental caries, dental fillings or restorations; 2) healthy periodontal tissue: plaque index and gingival index $\leq 1$, without obvious gingival recession or periodontal disease history; 3 ) no sleep bruxism history, no attrition of full dentition (attrition score $\leq 2)$; 4) normal or incomplete overbite and overjet; 5) normal occlusal curve; 6) no administration of gingival hyperplasia-related medicines within the latest 3 months; 7) no obvious gingival color pigmentation; and 8) age: $18-40$ years.

\section{D digital model construction}

The maxillary casts of the study participants were scanned using an intra-oral scanner and the STL format model files were numbered \#1-\#56. The *.STL files were imported into SpaceClaim software to generate 3D digital models (Fig. 1).

\section{Determination of the reference plane and esthetic landmarks}

The reference plane and esthetic landmarks were identified as previously described [16]. Briefly, for the description of the positional relationship between the maxillary dentition and gingival landmarks, an occlusal plane was selected as the horizontal reference plane, with 22 esthetic landmarks including the gingival zenith and the vertical bisected middle surface along the long axis of the clinical crown (VBMS). The intersection between the VBMS and gingival margin at the labial side was marked as the midpoint (Fig. 2).

\section{CGM index and measurement (accuracy: $0.01 \mathrm{~mm}$ )}

CGM indexes were measured according to specific definitions as follows:

- Gingival angle (GA): the angle between the gingival zenith at the labial side and the top of the corresponding mesial and distal papilla [9] (Fig. 2c).

- Papilla width (PW): the distance between the gingival zeniths of the two adjacent teeth. Each

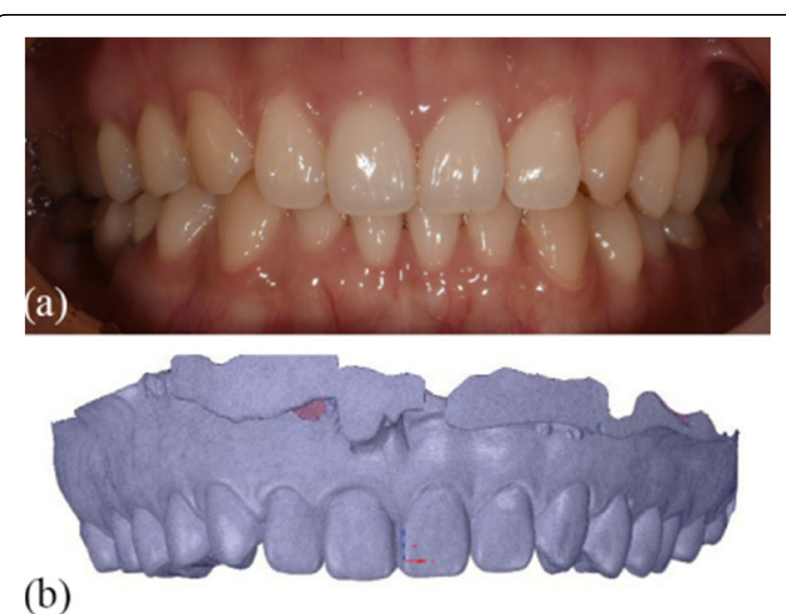

Fig. 1 Intra-oral image and 3D digital models. a Intra-oral image (b) $3 \mathrm{D}$ digital model 


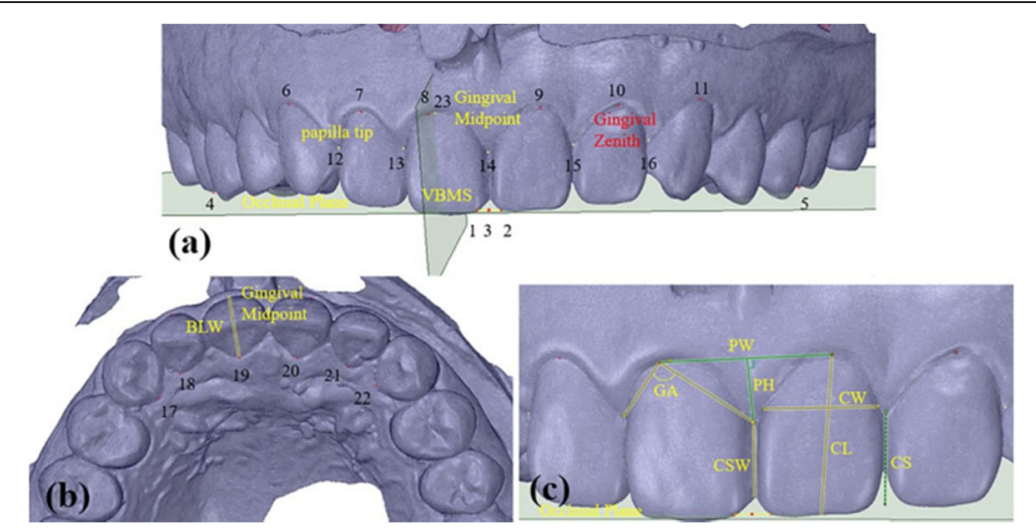

Fig. 2 Esthetic-related landmarks (a) Landmarks 1 and 2, the mesio-incisal angle of the maxillary central incisors on both sides; Landmark 3, the midpoint of the mesio-incisal angle ligature between the maxillary central incisors on both sides; Landmarks 4 and 5, the mesio-buccal cusp tip of the maxillary right and left first molars; Landmarks 6-11, the gingival edge apical top of the MAT on the labial side; Landmark 12-16, the top of the gingival papilla of the MAT; Landmark 23, the midpoint of the right maxillary central incisors at the gingival edge midpoint. $\mathbf{b}$ Landmarks 17-22, the top at the direction of the gingival edge of the MAT on the palate side

value was then associated with the mesially positioned tooth (Fig. 2c).

- Papilla height (PH): the shortest distance from the top of the papilla to the segment PW [14]. The mesial PH was recorded for every tooth position (Fig. 2c).

- Crown length (CL): the distance from the gingival zenith to the midpoint of incisal edge (or dental cusp). (Fig. 2c).

- Crown width $(\mathrm{CW})$ : the distance between the approximal tooth surfaces was recorded at the border between the middle and cervical portions. (Fig. 2c).

- Bucco-lingual width of the crown (BLW): the distance from the gingival margin at the side of the palate side to the apex to the gingival midpoint on the labial side [9]. (Fig. 2b).

- Contact surface width (CSW): the distance between the contact areas of the most apical portion and the most incisal portion. The mesial CSW was recorded for every tooth position. (Fig. 2c).

- Contact surface height (CS): the shortest distance from the most apical point in the mesial contact area to the incisal edge [13]. The mesial contact area height was recorded for every tooth position, and CS/CL value was calculated (Fig. 2c).

All measurement data were obtained by the same clinical researcher. After an interval of 1 month, six samples were randomly selected from the master samples for remeasurement.

\section{Evaluation of PBs based on the transparency of the periodontal probe through the gingival sulcus} Study participants lay in the supine position on the treatment chair, with the occlusal plane perpendicular to the ground. After the area of the MAT was dried and a black background put into the mouth, the standard Williams periodontal probe (KPW, Shanghai Kangqiao Dental Instruments Factory, Shanghai, China) was placed into the sulcus at the midfacial aspect of the right maxillary central incisor. The periodontal probe was placed parallel to the long axis of the clinical crown at a probing depth of $1 \mathrm{~mm}$ (the probe would reach to the bottom of the gingival sulcus at a probing depth $<1 \mathrm{~mm}$ ) [17]. A digital camera (Nikon D750, Nikon Corporation, Tokyo, Japan) equipped with a Microspur $105 \mathrm{~mm}$ lens (AF-S VR $105 \mathrm{~mm} \mathrm{f/2.8G} \mathrm{IF-ED,} \mathrm{Nikon} \mathrm{Corporation)}$ and a Microspur flashlight (Nikon R1C1, Nikon Corporation) was used to take photos in a standardized manner as follows: 1) unified shooting conditions with respect to factors such as light, background, and distance; 2) adoption of the same camera setting parameters; and 3) the teeth evaluated were placed at the center of photos, which should include around two natural teeth. All the examinations were completed by the same postgraduate student, and all photos were obtained by the same nurse.

The photos of all experimental subjects were randomly placed into the PPT file (Microsoft). After training and alignment by examiners, three postgraduates independently conducted qualitative periodontal biotyping according to the following standards for PB classification $[5,10,14,18,19]: 1)$ for thin biotypes, the probe was visible through the gingival tissue when placed within the gingival sulcus (Fig. 3a); 2) for thick biotypes, the probe was not visible through the gingival tissue when placed within the gingival sulcus (Fig. 3b).

After an interval of 2 weeks, the qualitative periodontal biotyping repeated independently, with the aim of lowering the bias in the first assessment. The determination 


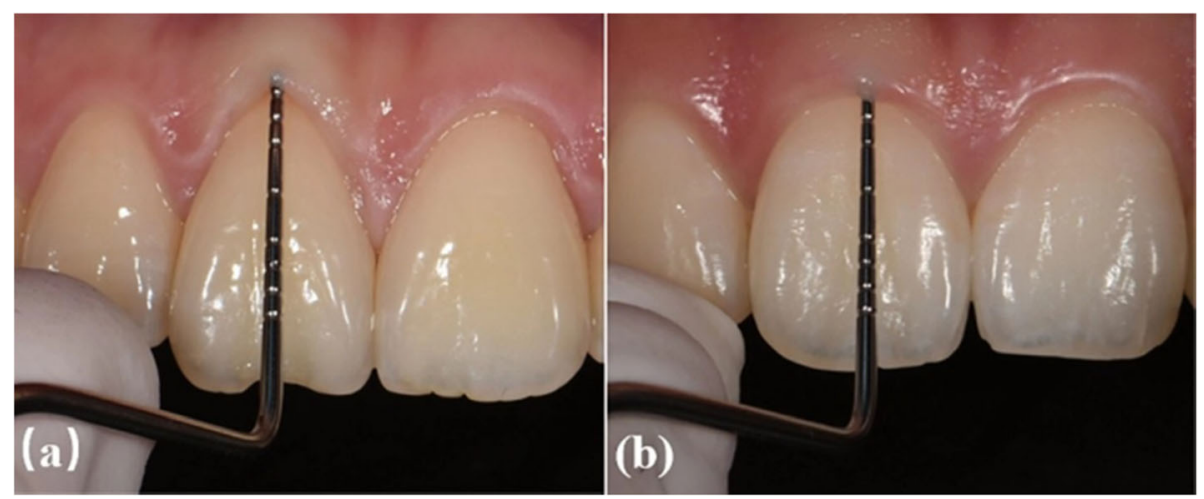

Fig. 3 Periodontal biotype. a Probe visible through marginal tissue; thin biotype. b Probe not visible through marginal tissue; thick biotype

of PBs for every study participant was based on consistent biotyping by two out of three examiners, with Kappa adopted to verify the reliability.

\section{Statistical analysis}

For all the continuous variables (e.g., GA and PW), the test-retest reliability of the examiners was evaluated through the Pearson correlation coefficient test using SPSS Statistics 24.0 software (IBM, Chicago, USA), while the intra-examiner repeatability was evaluated with the Kappa test. In addition, the Shapiro-Wilks test was used to verify the normal distribution of data. The average values of clinical parameters were further analyzed by verifying that there was no significant difference in measurements of clinical parameters of the teeth at the same position on both sides of the mouth using the Wilcoxon signed-rank test and paired sample $t$-test. The normal distribution of data was confirmed, with measurement data expressed in mean \pm standard deviation (SD). Analysis of variance (ANOVA) was used to evaluate differences between groups. Enumeration data were expressed as frequencies, and the chi-square test $\left(\chi^{2}\right.$ test) was used to evaluate differences between groups. PB was assigned as a dependent variable, while the factors with ANOVA $P \leq 0.2$ were assigned as independent variables. Forward: LR method was used for logistic regression to study the independent influence factors of PBs (inclusion equation standard 0.05, elimination standard 0.10). ROC curve analysis was repeated to evaluate the value of the factors that were finally entered into the logistic regression model for the diagnosis of PBs. P-values $<0.05$ were considered to indicate statistical significance.

\section{Results}

Test-retest reliability analysis showed relatively high reliability of all indexes $(r \geq 0.916, P<0.001)$, except the Pearson correlation coefficient in the $\mathrm{PH}$ associated with the mesial aspect of the maxillary canines $(r=0.657, P<$ 0.001 ), indicating the repeatability of the data. The average Kappa coefficient of the transparency of the periodontal probe through the gingival sulcus was 0.733 $(P<0.001)$, indicating the high degree of reliability of the evaluation method. The Wilcoxon signed-rank test and paired sample $t$-test. Results showed that there were no significant differences in the measurements of clinical parameters such as GA and PW for the teeth at the same location and interdental position on both sides of the mouth $(P \geq 0.069)$.

The CGM characteristics of the MAT of the study participants are as shown in Table 1.

The periodontal biotyping of the study participants is shown in Table 2. Our results showed that among the thick biotype accounted for the largest proportion (69.6\%) of the 56 study participants.

The characteristic parameters of periodontal biotypes of different genders are shown in Table 3. ANOVA test showed that there were statistical differences in periodontal biotypes between different genders. $(P \leq 0.2)$.

For different PBs, the CGM characteristics of the right maxillary central incisors are shown in Table 4. ANOVA showed that there were significant differences among the PBs in terms of in GA, PW, PH, CW, CW/CL and

Table 1 The CGM characteristics of MAT

\begin{tabular}{llll}
\hline Factor & $\begin{array}{l}\text { Central incisor } \\
\text { Mean } \pm \text { SD }\end{array}$ & $\begin{array}{l}\text { Lateral incisor } \\
\text { Mean } \pm \text { SD }\end{array}$ & $\begin{array}{l}\text { Canine } \\
\text { Mean } \pm S D\end{array}$ \\
\hline $\mathrm{GA}\left({ }^{\circ}\right)$ & $98.19 \pm 7.69$ & $96.24 \pm 10.03$ & $89.45 \pm 6.63$ \\
$\mathrm{PW}(\mathrm{mm})$ & $10.05 \pm 0.79$ & $7.83 \pm 0.60$ & $7.97 \pm 0.65$ \\
$\mathrm{PH}(\mathrm{mm})$ & $3.65 \pm 0.59$ & $3.37 \pm 0.53$ & $3.28 \pm 0.57$ \\
$\mathrm{CL}(\mathrm{mm})$ & $9.53 \pm 0.77$ & $8.26 \pm 0.77$ & $9.09 \pm 0.79$ \\
$\mathrm{CW}(\mathrm{mm})$ & $7.51 \pm 0.62$ & $5.84 \pm 0.48$ & $6.64 \pm 0.52$ \\
$\mathrm{CW} / \mathrm{CL}$ & $0.791 \pm 0.077$ & $0.713 \pm 0.081$ & $0.735 \pm 0.070$ \\
$\mathrm{BLW}(\mathrm{mm})$ & $7.22 \pm 0.53$ & $6.56 \pm 0.52$ & $8.38 \pm 0.48$ \\
$\mathrm{CSW}(\mathrm{mm})$ & $4.39 \pm 0.72$ & $3.56 \pm 0.56$ & $2.62 \pm 0.57$ \\
$\mathrm{CS} / \mathrm{CL}$ & $0.597 \pm 0.069$ & $0.623 \pm 0.070$ & $0.635 \pm 0.057$ \\
\hline
\end{tabular}


Table 2 The frequency distribution of PBS

\begin{tabular}{llll}
\hline & Male participants $(\mathrm{n})$ & Female participants $(\mathrm{n})$ & Total $[\mathrm{n}(\%)]$ \\
\hline Thin & 1 & 16 & $17(30.4)$ \\
Thick & 12 & 27 & $39(69.6)$ \\
Total & 13 & 43 & $56(100)$ \\
\hline
\end{tabular}

CS/CL $(P \leq 0.2)$, but no significant differences in terms of CL, BLW, and CSW $(P>0.2)$.

Table 5 presents the logistic regression results of multiple $\mathrm{PB}$ factors, showing that GA $(\mathrm{OR}=1.206, P=$ $0.016)$ and $\mathrm{PW}(\mathrm{OR}=5.048, P=0.002)$ were the independent influencing factors of $\mathrm{PB}$. The logistic regression model was used to re-categorize PBs, with a total accuracy of $85.7 \%$, as shown in Table 6 .

A ROC curve was generated with GA and PW as the test variables as shown in Fig. 4. The AUCs of GA and PW were 0.807 and 0.881 , respectively, and the optimal cutoff values of GA and PW were $95.95^{\circ}$ and $10.01 \mathrm{~mm}$, respectively. The combined AUC of GA and PW was 0.935, which was larger than the singular AUC for GA and PW, showing that the combined diagnosis of GA and PW contributes to increasing the diagnostic efficiency of PBs. In other words, when the GA and PW of the right maxillary central incisor are $95.95^{\circ}$ and 10.01 $\mathrm{mm}$, respectively, the optimal cutoff value for categorizing study participants as thick biotype, as shown in Table 7.

\section{Discussion}

In clinical practice, different PBs may react differently to inflammation and various types of dental treatment. Accurate pre-treatment diagnosis of the PB of patients is necessary to obtain the ideal effect. In this study, we conducted a quantitative analysis of the CGM morphology in terms of the clinical parameters of upper anterior teeth using 3D digital models with the aim of providing an accurate reference for the esthetic analysis and computer-aided design of anterior teeth, determining the cutoff value of gingiva and crown clinical parameters, and establishing clinical guidelines to offer quantitative guidance for periodontal biotyping.

Among the 56 participants included in this study, the thick biotype accounted for the largest proportion (69.6\%), while thin biotype accounted for only $30.4 \%$. Furthermore, there was statistically significant difference in PBs between males and females,

Table 3 Characteristics of periodontal biotypes in different genders

\begin{tabular}{|c|c|c|c|c|}
\hline Index & Thin & Thick & $X^{2} / F$ & P \\
\hline Gender & & & 4.281 & 0.043 \\
\hline
\end{tabular}

Table 4 The CGM characteristics of the right maxillary central incisors in different PBs

\begin{tabular}{lllll}
\hline Index & Thin & Thick & $X^{2} / F$ & $P$ \\
\hline GA $\left(^{\circ}\right)$ & $92.73 \pm 6.21$ & $101.68 \pm 8.03$ & 16.704 & 0.000 \\
PW (mm) & $9.43 \pm 0.53$ & $10.39 \pm 0.61$ & 31.955 & 0.000 \\
PH (mm) & $3.91 \pm 0.58$ & $3.53 \pm 0.56$ & 5.163 & 0.027 \\
CL (mm) & $9.69 \pm 0.81$ & $9.41 \pm 0.81$ & 1.428 & 0.237 \\
$\mathrm{CW}(\mathrm{mm})$ & $7.15 \pm 0.58$ & $7.67 \pm 0.61$ & 8.644 & 0.005 \\
$\mathrm{CW} / \mathrm{CL}$ & $0.736 \pm 0.065$ & $0.819 \pm 0.078$ & 14.465 & 0.000 \\
$\mathrm{BLW}(\mathrm{mm})$ & $7.16 \pm 0.53$ & $7.25 \pm 0.59$ & 0.264 & 0.61 \\
$\mathrm{CSW}(\mathrm{mm})$ & $4.33 \pm 0.70$ & $4.50 \pm 0.82$ & 0.55 & 0.462 \\
$\mathrm{CS} / \mathrm{CL}$ & $0.575 \pm 0.074$ & $0.611 \pm 0.074$ & 2.772 & 0.102 \\
\hline
\end{tabular}

as shown in Table 3, which is consistent with the results reported by De Rouck et al. [5]. However, Lee et al. [14] found that sex had no significant influence on PBs, with the thin biotype accounting for $21.8 \%$ of the individuals evaluated. In contrast, Frost et al. [18] reported that the thin biotype accounted for only $7 \%$ of their study participants. It can be speculated that sample size and ethnic differences may be the major factors contributing to the inconsistency in these results.

The contour of the gingival margin is determined by parameters such as the gingival angle, papilla width, and papilla height. The GA averages of the maxillary central incisor, lateral incisor and canines of all study participants were $98.19 \pm 7.69^{\circ}, 96.24 \pm 10.03^{\circ}$ and $89.45 \pm 6.63^{\circ}$, respectively. However, Olsson et al. [9] reported GAs for the maxillary central incisor, lateral incisor and canines of $86.60^{\circ}, 82.80^{\circ}$ and $80.29^{\circ}$, respectively. Differences in measurement methods may account for these inconsistencies. Olsson et al. [9] determined GA with the cosine function using intra-oral images to generated 3D digital models. This is a simple, convenient and accurate method of reflecting the spatial positional relation of the teeth and gingiva. The logistic regression model used in this study showed that GA $(P=0.016$, OR $=1.206)$ is an independent influencing factors of $\mathrm{PB}$. Our study also showed that the central incisor GAs of the thin and thick biotypes were $92.73 \pm 6.21^{\circ}$ and $101.68 \pm 8.03^{\circ}$, respectively. These results are consistent with those reported by Olsson [9] and Zhou Zhixuan et al. [4], suggesting that the GA of the thin biotype is smaller and the gingival margin more curved than that of the thick biotype.

Table 5 Multi-factor logistic regression of PB

\begin{tabular}{lllcl}
\hline $\begin{array}{l}\text { Influencing } \\
\text { factor }\end{array}$ & OR & $P$ & OR95\%Cl & \\
\cline { 4 - 5 } & & & Lower limit & Upper limit \\
\hline GA & 1.206 & 0.016 & 1.035 & 1.405 \\
PW & 5.048 & 0.002 & 2.705 & 83.710 \\
\hline
\end{tabular}


Table 6 Logistic regression prediction categorization of PBs

\begin{tabular}{lccl}
\hline Observed & \multicolumn{2}{l}{ Predicted } & \\
\cline { 2 - 4 } & Thin & Thick & Percentage correct \\
\hline Thin & 12 & 5 & 70.6 \\
Thick & 3 & 36 & 92.3 \\
Overall percentage & & & 85.7 \\
\hline
\end{tabular}

The morphology of the gingival papilla is a major evaluation index used in various current anterior teeth esthetic evaluation systems. The present study showed that the PWs of the maxillary central incisor, lateral incisor and canines in all the study participants were $10.05 \pm 0.79 \mathrm{~mm}, 7.83 \pm 0.60 \mathrm{~mm}$ and $7.97 \pm 0.65 \mathrm{~mm}$, respectively, which is consistent with the findings of Zhou Zhixuan et al. [4]. The logistic regression model of the right maxillary central incisor indicates that $\mathrm{PW}$ has a significant influence on $\mathrm{PB}(P=0.002, \mathrm{OR}=5.048)$, making the gingival papilla of the maxillary central incisor of the thin biotype narrower, although there are few studies on the correlation between PB and PW.

Olsson et al. [9] proposed that the PHs for the maxillary central incisor, lateral incisor and canines were 4.16 $\mathrm{mm}, 4.02 \mathrm{~mm}$ and $4.21 \mathrm{~mm}$, respectively, although the results of our study revealed values of $3.65 \pm 0.59 \mathrm{~mm}$, $3.37 \pm 0.54 \mathrm{~mm}, 3.28 \pm 0.57 \mathrm{~mm}$, respectively. This disparity may be attributed to the differences in study participants and measurement methods. In addition, ANOVA showed a significant difference $(P=0.027)$ between $\mathrm{PH}$ and $\mathrm{PB}$, while logistic multi-factor regression analysis suggested that $\mathrm{PH}$ is not an independent influencing factor of PB. De Rouck et al. [5] also reported a significant difference in $\mathrm{PH}$ between $\mathrm{PBs}$, while Olsson [9] and Stein [10] et al. claimed that there was no obvious correlation between gingival thickness and $\mathrm{PH}$. Lee et al. [14] found that the sum of five gingival papilla heights of the MAT larger than $24 \mathrm{~mm}$ was the identification standard for the thin biotype, and PB had no obvious correlation with the papillary height between two central incisors. The disparity in measurement methods and periodontal biotyping methods may be the major reasons for these differences.

Using image analysis software to calculate CW/CL, Stein et al. [10] found that CW/CL and PB were closely related, and therefore could be used to represent the predictive index for gingival thickness. In this study, ANOVA showed a significance difference between $\mathrm{CW} /$ $\mathrm{CL}$ and $\mathrm{PB}(P<0.001)$, although in logistic regression model, CW/CL was eliminated from the regression equation, indicating that it is not an independent influencing factor of $\mathrm{PB}$. This finding is consistent with those of Olsson [9] and Eger [20] and may be related to the difficulty in determining the most appropriate reference points, because CL is subject to the influence of attachment loss, gingival inflammation and incisal attrition, while CW is subject to the influence of the gingival papilla [9]. Moreover, differences in ethnicity and region may lead to different crown morphologies.

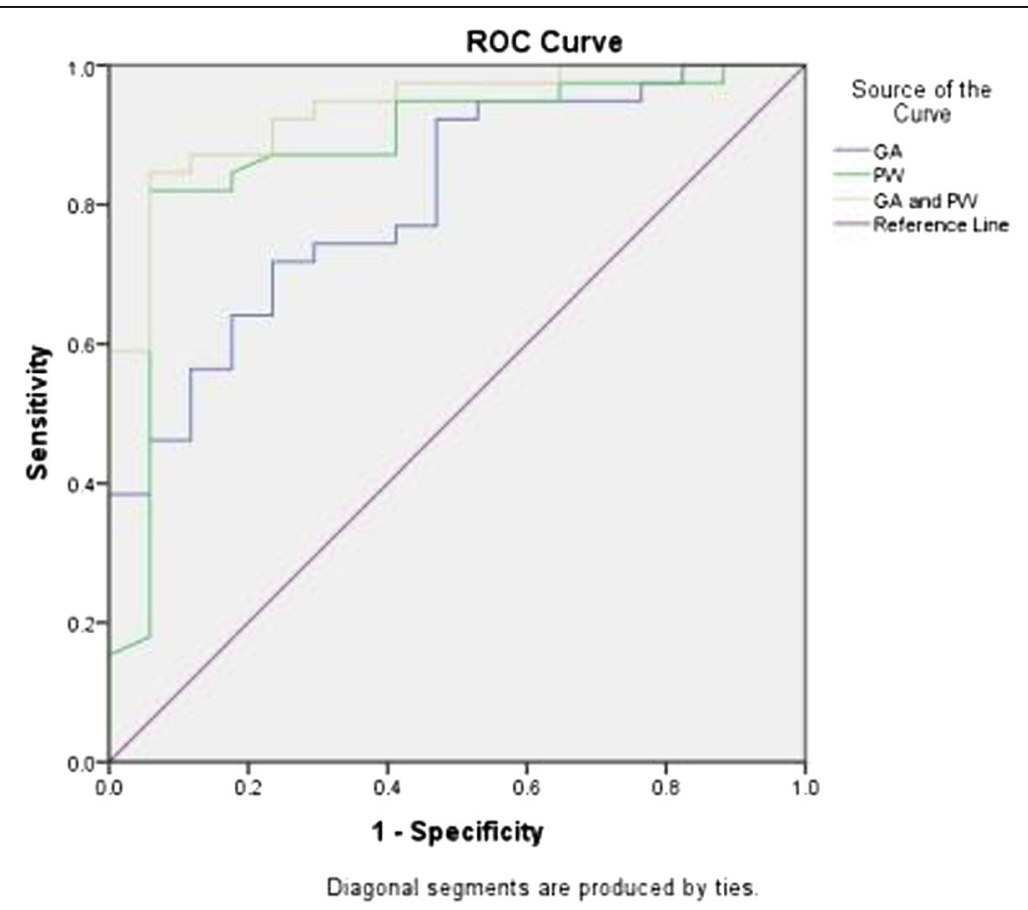

Fig. 4 ROC curve plotting sensitivity and specificity values to predict thick biotype at various cutoff values of GA and PW 
Table 7 Diagnostic value of gingival edge angle and papilla width of PBs

\begin{tabular}{lllllll}
\hline Variables & AUC & $95 \% \mathrm{Cl}$ & $P$ & Sensitivity (\%) & Specificity & Cutoff values \\
\hline GA $\left(^{\circ}\right)$ & 0.807 & $0.689-0.925$ & 0.000 & 71.8 & 76.5 & 95.95 \\
PW (mm) & 0.881 & $0.774-0.988$ & 0.000 & 82.1 & 94.1 & 10.01 \\
GA and PW & 0.935 & $0.871-0.999$ & 0.000 & 0.846 & 0.941 & 0.76 \\
\hline
\end{tabular}

$\mathrm{Cl}$ : Confidence Interval

By measuring casts, Olsson [9] reported BLWs of the maxillary central incisor, lateral incisor and canines of $7.33 \pm 0.56 \mathrm{~mm}, 6.51 \pm 0.57 \mathrm{~mm}$ and $8.29 \pm 0.65 \mathrm{~mm}$, respectively, indicating a significant correlation between gingival thickness and BLW. According to our study, the BLWs of the maxillary central incisor, lateral incisor and canines are $7.22 \pm 0.53 \mathrm{~mm}, 6.56 \pm 0.52 \mathrm{~mm}$, and $8.38 \pm$ $0.48 \mathrm{~mm}$, respectively, with no correlation between BLW and $\mathrm{PB}$ found. This discrepancy may be attributed to differences in the study participants and the PB diagnosis method.

Tarnow et al. [21] reported that the esthetic effect of the gingival papilla was associated with the position of the contact area. In this study, the CSWs of the maxillary central incisor, lateral incisor and canines were $4.39 \pm 0.72 \mathrm{~mm}, 3.56 \pm 0.56 \mathrm{~mm}$, and $2.62 \pm 0.57 \mathrm{~mm}$, respectively, and the CS/CL values were $0.597 \pm 0.069$, $0.623 \pm 0.070$ and $0.635 \pm 0.057$, respectively. Moreover, compared with thin biotype, the contact surface of the thick biotype is wider, and the most apical portion of the contact area is closer to the gingival margin, although no significant differences were found between PBs. Gobbato et al. [13] categorized the maxillary central incisors, finding that the most apical portion of the contact area in the triangular group was closer to the incisal edge, while that in the square group was closer to the gingival margin.

Most previous studies have focused on the correlation of $\mathrm{PB}$ with the morphology of the soft and hard tissues $[5-8,10-12,17,19,22-24]$, with little investigation of the influence of independent factors on PB diagnosis efficiency. The logistic regression results of our study show that the right central incisor GA and PW are important predictive factors of $\mathrm{PB}$, with the probability of a thick biotype diagnosis increasing 1.206 times for every $1^{\circ}$ increase in GA, while the probability of a thick biotype diagnosis increases 5.048 times for every I $\mathrm{mm}$ increase in PW. This supports the hypothesis that "compared with thick biotype, the free gingival margin at the labial side of the thin biotype is more curved and the gingival papilla narrower" $[5,9,25,26]$. We found that the GA, PW and combined AUC were 0.807, 0.881, and 0.935 , respectively, indicating that the combination of GA and PW improve the diagnostic efficiency of PB. In this study, the GA and PW of the right maxillary central incisors of $95.95^{\circ}$ and $10.01 \mathrm{~mm}$, respectively, were identified as the optimal cutoff values to categorize individuals as thick biotype. This implies an increased probability of categorizing individuals as thick biotype when the GA and PW of the right maxillary central incisors are $\geq 95.95^{\circ}$ and $\geq 10.01 \mathrm{~mm}$, respectively. In an analysis of the relationship between gingival thickness and PB based on ROC curves, Frost et al. [19] failed to identify a suitable gingival thickness threshold for diagnosis a thick biotype.

This exploration of CGM parameters and their correlation with $\mathrm{PB}$ using 3D digital models is limited by the small sample size, uneven sex ratio, and single focus on the correlation of the right maxillary central incisor $\mathrm{PB}$ with CGM clinical parameters. Therefore, in future studies, it is necessary to expand the sample size, balance the sex ratio, and take into consideration the correlation of the periodontal biotypes at different teeth positions with CGM. In addition, the limited number of influencing factors included in this research may have ignored the influence of other factors on PB; therefore, future investigations should evaluate the influence of factors such as alveolar bone morphology, keratinized gingival width and gingival thickness to provide more powerful evidence for the diagnosis of PBs in the clinic. In addition, randomized controlled trials are required to verify the potential of GA and PW for improving the accuracy of PB diagnosis.

\section{Conclusions}

With the occlusal plane as the reference, the CGM at both sides is symmetrical. The thin biotype accounts for a small proportion of cases; in which the free gingival margin at the labial side of the central incisor is more curved, and the gingival papilla narrower than those of the thick biotype. For a long-narrow crown, the buccolingual width of the crown is smaller, the contact surface is larger, and the most apical portion of the contact area is closer to the incisal edge. Moreover, GA and PW are independent influencing factors of the $\mathrm{PB}$ of the right maxillary central incisor. The GA and PW of the right maxillary central incisors of $95.95^{\circ}$ and $10.01 \mathrm{~mm}$, respectively, are the optimal cutoff values to categorize individuals as thick biotype. This implies an increased probability of categorizing individuals as thick biotype when the GA and PW of the right maxillary central incisors are $\geq 95.95^{\circ}$ and $\geq 10.01 \mathrm{~mm}$, respectively. 


\section{Abbreviations}

3D: Three-dimensional; AUC: Areas under the ROC curve; BLW: Bucco-lingual width of the crown; CAD/CAM: Computer-aided design/computer-aided manufacturing; CGM: Crown and gingival morphology; $\mathrm{CL}$ : Crown length; CS: Contact surface height; CS/CL: Contact surface height/crown length ratio; CSW: Contact surface width; CW: Crown width; CW/CL: Crown width/crown length ratio; GA: Gingival angle; MAT: Maxillary anterior teeth; OR: Odds ratio; PB: Periodontal biotype; PH: Papilla height; PW: Papilla width; ROC curve: Receiver operating characteristic curve; VBMS: Vertical bisected middle surface along the long axis of the clinical crown

\section{Acknowledgments}

The authors are grateful to Mr. Fengyue Bai (CATARC Automotive Test Center (Tianjin) Co., Ltd.) for his technical support.

\section{Authors' contributions}

XJY and XPK participated in conception and design of the work, collecting data, and drafted the manuscript. BYW contributed to the data collection. YY, TZ and MYJ performed the statistical analysis and participated in its design. XYL participated in collecting data. HQS participated in the acquisition, analysis, and interpretation of data and drafted the manuscript. All authors made a substantial contribution to this study and/or manuscript, and all have reviewed the final paper prior to its submission. The author(s) read and approved the final manuscript.

\section{Funding}

This study was supported by a grant from the Key Research and Development Program of Shandong Province (2019GSF107001). The funding bodies were not otherwise involved in the design of the study, nor the collection, analysis or interpretation of data and writing of the manuscript.

\section{Availability of data and materials}

The datasets used and/or analyzed during the current study are available from the corresponding author on reasonable request.

\section{Ethics approval and consent to participate}

This study was conducted in full accordance with the World Medical Association Declaration of Helsinki and was approved by the Medical Ethics Committee of the Affiliated Stomatological Hospital to Shandong University (No. R20180502). Written informed consent was obtained from all study participants.

\section{Consent for publication}

Not applicable.

\section{Competing interests}

The authors declare that they have no competing interests.

\section{Received: 10 August 2019 Accepted: 7 February 2020}

\section{Published online: 19 February 2020}

\section{References}

1. Seibert J, Lindhe J. Esthetics and periodontal therapy. In: Lindhe J, Karring T, editors. Textbook of clinical periodontology. 2nd ed. Copenhagen: Munksgaard; 1989. p. 477-513.

2. Eugenio R, Diego L, Alessandro R, Stefano S, Roberto R, Matteo C. Surgical and prosthetic management of interproximal region with single-implant restorations: 1-year prospective study. J Periodontol. 2008:79(6):1048-55.

3. Fu JH, Yeh CY, Chan HL. TatarakisnN., Leong DJ, Wang HL. Tissue biotype and its relation to the underlying bone morphology. J Periodontol. 2010; 81(4):569-74.

4. Zhou ZX, Shen M, Lu SN, Li J, Chen N. Comparative study on the morphology of crown, alveolar ridge crest and gingival in maxillary anterior region. Chin J Stomatol. 2013;48(4):211-5.

5. De Rouck T, Eghbali R, Collys K, De Bruyn H, Cosyn J. The gingival biotype revisited: transparency of the periodontal probe through the gingival margin as a method to discriminate thin from thick gingiva. J Clin Periodontol. 2009;36(5):428-33.

6. Fischer KR, Künzlberger A, Donos N, et al. Gingival biotype revisited-novel classification and assessment tool. Clin Oral Investig. 2018;22(1):443-8.
7. Muller HP, Eger T. Gingival phenotypes in young male adults. J Clin Periodontol. 1997:24(1):65-71.

8. Muller HP, Heinecke A, Schaller N, Eger T. Masticatory mucosa in subjects with different periodontal phenotypes. J Clin Periodontol. 2000;27(9):621-6.

9. Olsson M, Lindhe J, Marinello CP. On the relationship between crown form and clinical features of the gingiva in adolescents. J Clin Periodontol. 1993; 20(8):570-7.

10. Stein JM, Lintel-Hoping N, Hammacher C, Kasaj A, Tamm M, Hanisch O. The gingival biotype: measurement of soft and hard tissue dimensions - a radiographic morphometric study. J Clin Periodontol. 2013;40(12):1132-9.

11. Xie GY, Chen JH. Cluster analysis for gingival morphological characteristics of maxillary anterior tooth segment in Han Chinese. Chi J Aesthet Med. 2007;16(3):380-2.

12. Alves PHM, Alves TCLP, Pegoraro TA, Costa YM, Bonfante EA, de Almeida ALPF. Measurement properties of gingival biotype evaluation methods. Clin Implant Dent Relat Res. 2018;20(3):280-4.

13. Gobbato L, Tsukiyama T, Levi PA Jr, Griffin TJ, Weisgold AS. Analysis of the shapes of maxillary central incisors in a Caucasian population. Int J Periodontics Restorative Dent. 2012;32(1):69-78.

14. Lee SP, Kim TI, Kim HK, Shon WJ, Park YS. Discriminant analysis for the thin periodontal biotype based on the data acquired from three-dimensional virtual models of Korean young adults. J Periodontol. 2013:84(11):1638-45.

15. Wong NK, Kassim AA, Foong KW. Analysis of esthetic smiles by using computer vision techniques. Am J Orthod Dentofac Orthop. 2005;128(3): 404-11.

16. Ke XP, Wang CW, Sun HQ, Yang Y, Luo XY, Liu TS. A quantitative research on clinical parameters of gingival contour for anterior teeth esthetic analysis and design. J Esthet Restor Dent. 2018;30(6):532-7.

17. Le D, Zhang H, Hu WJ, Liu DG. Preliminary study on gingival biotype by periodontal probing. Chin J Stomatol. 2012;47(2):81-4.

18. Frost NA, Mealey BL, Jones AA, Huynh-Ba G. Periodontal biotype: gingival thickness as it relates to probe visibility and Buccal plate thickness. J Periodontol. 2015:86(10):1141-9.

19. Kan JY, Rungcharassaeng K, Umezu K, Kois JC. Dimensions of peri-implant mucosa: an evaluation of maxillary anterior single implants in humans. $J$ Periodontol. 2003;74(4):557-62.

20. Eger T, Müller HP, Heinecke A. Ultrasonic determination of gingival thickness. Subject variation and influence of tooth type and clinical features. J Clin Periodontol. 1996;23(9):839-45.

21. Tarnow DP, Magner AW, Fletcher P. The effect of the distance from the contact point to the crest of bone on the presence or absence of the interproximal dental papilla. J Periodontol. 1992;63(12):995-6.

22. Eghbali A, De Rouck T, De Bruyn H, Cosyn J. The gingival biotype assessed by experienced and inexperienced clinicians. J Clin Periodontol. 2009:36(11): 958-63.

23. Kan JY, Morimoto T, Rungcharassaeng K, Roe P, Smith DH. Gingival biotype assessment in the esthetic zone: visual versus direct measurement. Int J Periodontics Restorative Dent. 2010;30(3):237-43.

24. Muller HP, Kononen E. Variance components of gingival thickness. J Periodontal Res. 2005:40(3):239-44.

25. Shu R. The influence of periodontal biotype on multidisciplinary treatment of oral cavity. Chinese Journal of Stomatology. 2014;49(3):129-32.

26. Olsson $\mathrm{M}$, Lindhe J. Periodontal characteristics in individuals with varying form of the upper central incisors. J Clin Periodontol. 1991;18(1):78-82

\section{Publisher's Note}

Springer Nature remains neutral with regard to jurisdictional claims in published maps and institutional affiliations. 